Research Article

\title{
Experimental Study on the Dynamic Rock-Breaking Performance of Pulsed Abrasive Jet Drilling Method
}

\author{
Yukun Du $\left(\mathbb{D},{ }^{1,2}\right.$ Xiaohong Chen $\left(\mathbb{D},{ }^{2}\right.$ Bo Zhao, ${ }^{3}$ Zhiyuan Huang, ${ }^{4}$ Meilian $W u,{ }^{5}$ \\ Diangang Bai, ${ }^{6}$ Guoliang Hao, ${ }^{7}$ Qingshi Meng, ${ }^{8}$ Zhenhua Mei, ${ }^{9}$ and Xiaocheng Chen ${ }^{8}$ \\ ${ }^{1}$ Key Laboratory of Unconventional Oil \& Gas Development, China University of Petroleum (East China), Ministry of Education, \\ Qingdao 266580, China \\ ${ }^{2}$ School of Petroleum Engineering, China University of Petroleum, Qingdao 266580, China \\ ${ }^{3}$ Oil Industry Training Centre, China University of Petroleum, Qingdao 266580, China \\ ${ }^{4}$ Drilling Technology Research Institute of Shengli Petroleum Engineering Corporation Limited, Sinopec, Dongying 257000, China \\ ${ }^{5}$ Lunnan Oil and Gas Development Department, CNPC Tarim Oilfield, Korla 841000, China \\ ${ }^{6}$ Seabeach Development Company, 4th Oil Production Plant of CNPC Dagang Oilfield, Tianjin 300280, China \\ ${ }^{7}$ Huanxiling Oil Production Plant, CNPC Liaohe Oilfield, Panjin 124114, China \\ ${ }^{8}$ Qingdao Geological Exploration of China Metallurgical Geology Bureau, Qingdao 266000, China \\ ${ }^{9}$ Geological Exploration Institute of Shandong Zhengyuan, Qingdao 250000, China
}

Correspondence should be addressed to Xiaohong Chen; 20130043@upc.edu.cn

Received 31 July 2020; Revised 14 September 2020; Accepted 3 October 2020; Published 16 October 2020

Academic Editor: Qilong Xue

Copyright (c) 2020 Yukun Du et al. This is an open access article distributed under the Creative Commons Attribution License, which permits unrestricted use, distribution, and reproduction in any medium, provided the original work is properly cited.

The efficient development of deep oil and gas reservoirs with abundant resources is conducive to meet the growing energy demand. However, it is very difficult to drill in the deep reservoirs such as tight sand gas and shale gas because of their high strength, low porosity, and low permeability. In this study, it is pointed out that developing high-efficiency drilling methods based on new combined water jets is a good approach to promote the rate of penetration (ROP) in such tight deep reservoirs. A pulsed abrasive water jet drilling tool is designed, and its dynamic work principle is analyzed. The hydraulic structure is optimized; meanwhile, the rock-breaking experiments of this structure are carried out. The results show that the rock-breaking performance of the pulsed water jet is much better than that of the continuous water jet. It is also found that the rock-breaking performance of the pulsed abrasive water jet is much better than that of the premixed abrasive water jet. In addition, the best rock-breaking standoff distance, abrasive concentration, and particle size are detected.

\section{Introduction}

The efficient development of deep reservoirs with abundant resources is conducive to meet the growing energy demand. However, most of the deep oil and gas in China widely exist in wells with poor drillability and low rock-breaking efficiency. Nowadays, the main production areas of deep oil and gas reservoirs in China lie in the western Tarim, Junggar, Qaidam, Turpan-Hami, and northeastern of Sichuan. Accidents are often encountered during drilling for the oil and gas reservoirs that are buried very deep [1]. This leads to the frequent occurrence of bottom-hole complex accidents, long-term construction cycle, and high cost. Therefore, an effective deep and ultradeep drilling technology should increase the penetration rate, shorten the cycle, and drill safely while reducing cost. In order to achieve this goal, there is an urgent need to develop new technologies to promote the ROP in deep complicated formations.

In contrast to some common technologies, it is proved practically that reasonably using the hydraulic energy or hydraulic energy-driven bottom-hole tools for rock breaking is a feasible way to promote the ROP in deep formations without increasing the device capabilities on the ground. Using the high-pressure water jet for rock breaking is an 
important way of reasonably using the bottom-hole hydraulic energy [2-6]. Particle impact drilling technology is a successful abrasive jet drilling technology; it is a high-efficiency noncontact rock-breaking process which is mainly based on subsonic particle impact and breaks rock, while being supplemented by the high-velocity water jet and mechanical bit [7]. The pulsed jet can reduce the pressure holding effect of bottom-hole rock, improve the stress state of rock, and enhance the effect of rock breaking and rock cleaning [8]. Both the abrasive water jet and pulsed water jet have distinctive characteristics [9-15], using some appropriate modulation methods can integrate their rockbreaking advantages and get a much better effect than using any of them alone.

In this study, the technology advantages of the pulsed water jet and abrasive water jet are integrated and a kind of pulsed abrasive water jet drilling tool is put forward. The pulsed abrasive jet drilling technology uses drilling cuttings as an abrasive medium to form the pulsed abrasive jet acting on bottom-hole rock. It does not change the existing drilling process but only needs to install a short joint between the drill collar and bit. Therefore, the technology is very suitable for the practical application. The hydraulic structure of this tool is optimized by the orthogonal experimental method, the dynamic rock-breaking performance of the pulsed abrasive jet is analyzed, and the best rock-breaking standoff distance, abrasive concentration, and particle size are detected.

\section{Experimental Preparation}

2.1. Materials. In this paper, white quartz sand with sharp edges and corners bought from the Research Institute of Petroleum Engineering of Shengli Oil Field of Sinopec is selected as the abrasive particles to replace drilling cuttings. The size range of quartz sand is selected from $0.3 \mathrm{~mm}$ to $0.6 \mathrm{~mm}$ according to the field cuttings. The content of quartz in the white quartz sand is up to $99 \%$, and the density is $3100 \mathrm{~kg} / \mathrm{m}^{3}$. The common Class G oil well cement and quartz sand are used to make testing rocks with the mass ratio of 2.5 and 2.3, and the uniaxial compressive strength is $10 \mathrm{MPa}$ and $35 \mathrm{MPa}$, respectively.

2.2. Experimental Setup. The pulsed abrasive jet drilling tool is designed, as is shown in Figure 1. The dynamic work principle is that the drilling fluid is modulated through the mixing chamber of the hydraulic oscillator, and a high-speed pulsed water jet will be formed in the exit after the selfexcited oscillation process. An area of low pressure is formed near the drawing entrance of the mixing chamber, and the cuttings containing drilling fluid is drawn in through the drawing entrance, and then the mixed pulsed abrasive water jet is accelerated by the bit nozzles of the drill bit and impacts on the rocks [16-23].

2.3. Experimental Procedure. The experimental tool of the pulsed abrasive jet is shown in Figure 2. The key structures to be identified are the nondimensional lower nozzle diameter

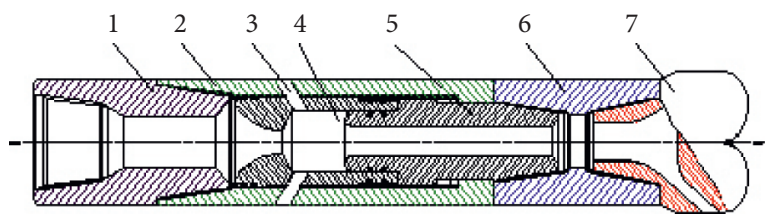

FIgURE 1: Self-excited pulsed abrasive jet drilling tool. (1) Upper connector; (2) body; (3) drawing entrance; (4) mixing chamber; (5) bit connecting rod; (6) lower connector; (7) drill bit.
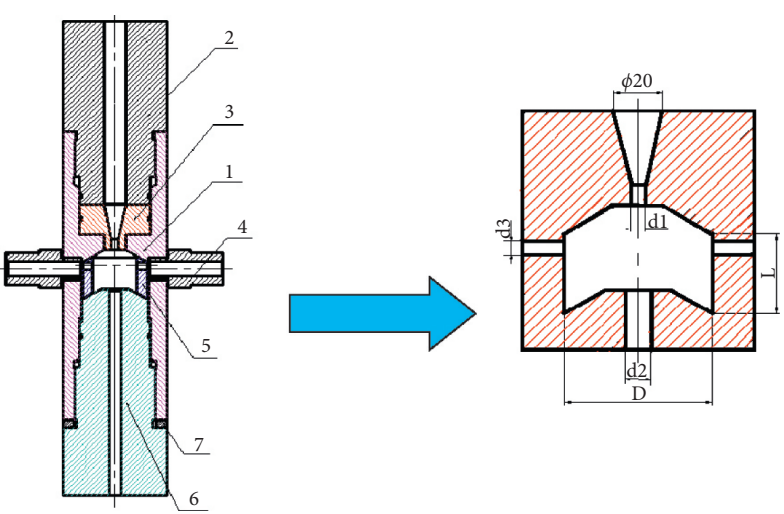

Figure 2: The experimental tool of the pulsed abrasive jet. (1) Body; (2) top connector; (3) upper nozzle; (4) drawing entrance; (5) sleeve; (6) lower nozzle; (7) gasket.

$d_{2}$, the nondimensional length of the oscillation cavity $L$, and the nondimensional diameter of the oscillation cavity $D$.

The common pulsed jet will be formed when the drawing entrance of the experimental tool is blocked, and the continuous jet will be formed when the lower nozzle is directly connected to the nozzle and there is no mixing chamber. The pulsed abrasive entrained water jet will be formed when the external fluid contains quartz sand and the drawing entrance of the experimental tool is open. Similar to particle jet drilling technology, the abrasive is added to the pipeline in the abrasive tank behind the plunger pump to form the premixed abrasive jet, rather than at the tool.

The rock-breaking experimental procedure is shown in Figure 3. (1) First, connect the water tank, plunger pump, safety valve, test bench, experimental tool, and relevant pipelines in accordance with the experimental procedure and place the test rock. (2) Fill the water tank and water box with water, put a certain amount of quartz sand in the water box, and stir fully. (3) Start the plunger pump; the pressurized fluid will run through the experimental tool and form the pulsed water jet. (4) Open the valve between the water box and the experimental tool after the pressure gauge reaches the predetermined value; the sand-containing fluid will be drawn into the tool and form the pulsed abrasive jet to impact the test rock. (5) Stop the plunger pump after reaching the predetermined test time, take out the rock, and test the rock-breaking effect of the jet on the test rock.

In this paper, we first carry out the orthogonal experiments to optimize the hydraulic parameters and determine a best rock-breaking structure of the pulsed abrasive water jet, and then this structure is used to compare the erosion 


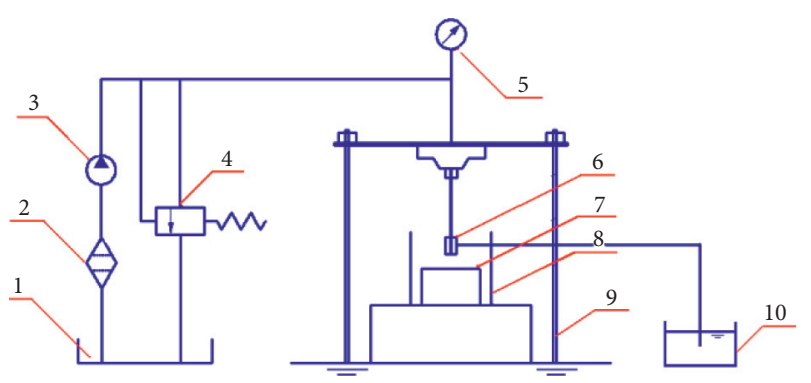

Figure 3: Experimental procedure of the pulsed abrasive water jet. (1) Water tank; (2) filter; (3) plunger pump; (4) safety valve; (5) pressure gage; (6) experimental tool; (7) rock; (8) iron box; (9) test bench; (10) water box (pure water or abrasive-containing water).

performance of the continuous water jet and usual pulsed water jet with that of the pulsed water jet while drawing in external water. Finally, another set of experiments are carried out to compare the erosion performance of the premixed abrasive water and pulsed premixed abrasive water jet with that of the pulsed abrasive entrained water jet of this optimized structure.

\section{Optimization Experiments of the Hydraulic Structure}

Pure water is used as the test medium. The testing rocks are made of quartz sand and common oil well cement with the mixed cement-sand ratio of $1: 2.3$, and the compressive strength is $35 \mathrm{MPa}$. The pump pressure is $15 \mathrm{MPa}$.

Set the diameter of the upper nozzle $d_{1}$ as $5 \mathrm{~mm}$ and the diameter of the drawing entrance $d_{3}$ as $6 \mathrm{~mm}$ on the basis of previous experiments [24]. In addition to the upper nozzle diameter $d_{1}$, there are five other factors which can affect the rock-breaking efficiency of the jet: the lower nozzle diameter $d_{2}$, the diameter of the oscillation cavity $D$, the length of the oscillation cavity $L$, the jet standoff distance $h$, and the abrasive concentration. Choosing the $5 \mathrm{~mm}$ upper nozzle diameter as the reference dimension, the nondimensional data will be obtained by using other parameter dimensions comparing with it. The orthogonal experimental method is used to comprehensively analyze the effect of these five factors on the rock-breaking efficiency of the experimental tool. Each factor has four levels, as is shown in Table 1.

According to the factors and the levels in Table 1, the orthogonal experimental design table is made. In order to reduce the experiment amount, the $L_{16}\left(4^{5}\right)$ orthogonal table is chosen. 16 group tests have been carried out, and the results are shown in Table 2.

By variance analysis of the orthogonal experimental results, we can see that the lower nozzle diameter and the length of the oscillation cavity are major factors affecting the rock-breaking efficiency of the jet. The optimized structure of the experimental tool is $\mathrm{A}_{2} \mathrm{~B}_{1} \mathrm{C}_{1} \mathrm{D}_{1} \mathrm{E}_{4}$, namely: the nondimensional lower nozzle diameter $d_{2}$ is 1.8 , the nondimensional length of the oscillation cavity $L$ is 6 , the nondimensional diameter of the oscillation cavity $D$ is 9 , the nondimensional jet standoff distance $h$ is 3 , and the abrasive concentration is $5 \%$.
The rock-breaking experiment is carried out with this structure. The erosion volume is $19 \mathrm{~cm}^{3}$, much better than the results of the front 16 group tests, so it is proved to be the best rock-breaking structure of the pulsed abrasive water jet. This set of structure is used in the subsequent rock-breaking experiments.

\section{Results and Discussion}

4.1. Rock-Breaking Experiments with Pure Water. We use pure water as the test medium. The testing rocks are made of quartz sand and common oil well cement with the mixed cement-sand ratio of $1: 2.5$, and their compressive strength is $10 \mathrm{MPa}$.

4.1.1. Benchmark Experiments. Figures 4 and 5 give the effect comparison of jet standoff distance and pressure on the erosion performance of the continuous water jet, usual pulsed water jet, and pulsed water jet while drawing in external water. The erosion volume first increases and then decreases with the increase in the jet standoff distance of the three kinds of water jets. Meanwhile, the erosion performance improves with the increase in pump pressure of all the three kinds of water jets. The rock-breaking performance of the pulsed water jet is much better than that of the continuous water jet. While the fluid entrance is open and the external water is drawn in, the rock-breaking performance of the pulsed water jet will be improved significantly.

4.1.2. Experimental Factors. The erosion volume first increases and then decreases with the increase in jet standoff distance (Figure 4). The reason is that when the jet standoff distance is shorter, the pulsed water jet will not fully develop; when the jet standoff distance increases appropriately, the jet turns to fully develop, so the impact area and erosion volume will increase. However, when the jet standoff distance goes on increasing, the energy consumption will increase rapidly and meanwhile the erosion volume will reduce correspondingly. While drawing in external water, the best jet standoff distance of the pulsed water jet is $15 \mathrm{~mm}$.

Figure 5 shows that the erosion performance improves with the increase in pump pressure just like a straight line because at this moment, water is accelerated in the entire high-pressure pipeline, so the impact energy of the water jet increases, as well as the breaking performance of the water jet on the rocks.

Table 3 gives a comparison of different drawing types of the external water on the rock-breaking performance. The more drawing entrances are opened, the more external fluid is drawn in, not that a better rock-breaking performance will be obtained, because at the same time, more hydraulic energy will be consumed. If we can draw in the external water as much as possible with proper drawing entrances, a much better rock-breaking performance will be obtained. In this experiment, the rock-breaking performance of the pulsed water jet with even numbers of drawing entrances is better than that of odd numbers, which is a bit better when the drawing entrances are placed symmetrically. It can be 
TABLE 1: The factors and levels of the orthogonal experiment.

\begin{tabular}{|c|c|c|c|c|}
\hline \multirow{2}{*}{ Levels } & \multicolumn{4}{|c|}{ Factors } \\
\hline & 1 & 2 & 3 & 4 \\
\hline A: the nondimensional lower nozzle diameter & 1.6 & 1.8 & 2 & 2.2 \\
\hline B: the nondimensional length of the oscillation cavity & 6 & 7 & 8 & 9 \\
\hline C: the nondimensional diameter of the oscillation cavity & 9 & 10 & 11 & 12 \\
\hline D: the nondimensional jet standoff distance & 3 & 4 & 5 & 6 \\
\hline E: the abrasive concentration $/ \%$ & 0.5 & 1 & 2 & 5 \\
\hline
\end{tabular}

TABLE 2: The orthogonal experimental results.

\begin{tabular}{lcccccc}
\hline \multirow{2}{*}{ Number } & \multicolumn{6}{c}{ Factors } \\
& A & B & C & D & E & Erosion volume $\left(\mathrm{cm}^{3}\right)$ \\
\hline 1 & 1.6 & 6 & 9 & 3 & 0.5 & 6 \\
2 & 1.6 & 7 & 10 & 4 & 1 & 0.8 \\
3 & 1.6 & 8 & 11 & 5 & 2 & 0.2 \\
4 & 1.6 & 9 & 12 & 6 & 5 & 2.5 \\
5 & 1.8 & 6 & 10 & 5 & 5 & 11.375 \\
6 & 1.8 & 7 & 9 & 6 & 2 & 9.5 \\
7 & 1.8 & 8 & 12 & 3 & 1 & 7.75 \\
8 & 1.8 & 9 & 11 & 4 & 0.5 & 3.75 \\
9 & 2 & 6 & 11 & 6 & 1 & 8.25 \\
10 & 2 & 7 & 12 & 5 & 0.5 & 4.6 \\
11 & 2 & 8 & 9 & 4 & 5 & 5 \\
12 & 2 & 9 & 10 & 3 & 2 & 7.5 \\
13 & 2.2 & 6 & 12 & 4 & 2 & 6.25 \\
14 & 2.2 & 7 & 11 & 3 & 5 & 6.75 \\
15 & 2.2 & 8 & 10 & 6 & 0.5 & 1.35 \\
16 & 2.2 & 9 & 9 & 5 & 1 & 3.05 \\
\hline
\end{tabular}

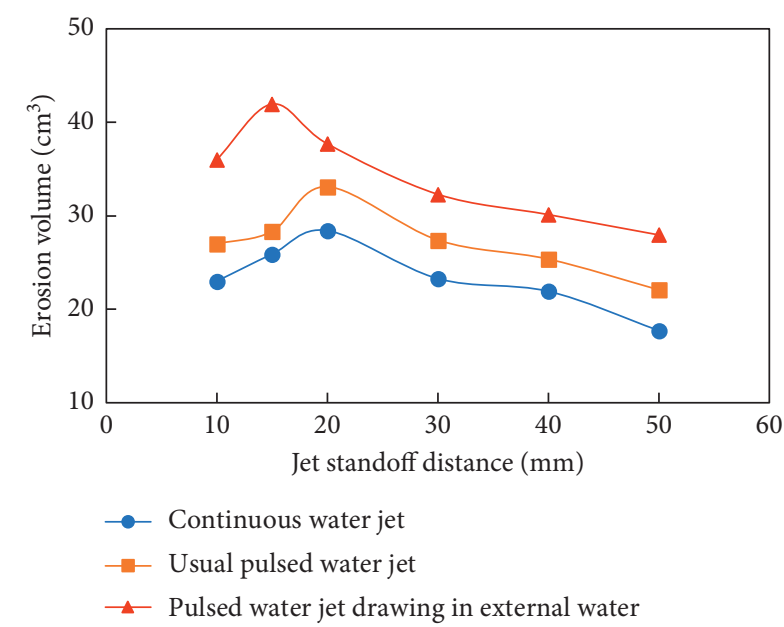

FIGURE 4: Effect of jet standoff distance on the erosion performance (when the pump pressure is $15 \mathrm{MPa}$ ).

seen that the proposal of two symmetric drawing entrances is the best drawing type in this experiment.

\subsection{Rock-Breaking Experiments of Pulsed Water Jet Mixed} with Abrasive. Quartz sand is selected as the abrasive medium and mixed in water. The testing rocks are made of quartz sand and common oil well cement with the mixed

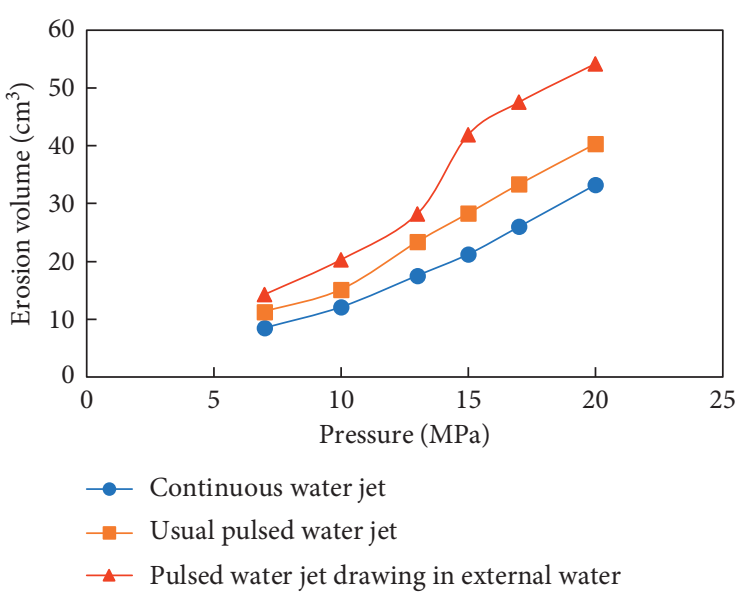

FIGURE 5: Effect of pump pressure on the erosion performance (when the jet standoff distance is $15 \mathrm{~mm}$ ).

TABLE 3: Effect of the drawing type on the erosion performance.

\begin{tabular}{lc}
\hline Drawing type & Erosion volume $\left(\mathrm{cm}^{3}\right)$ \\
\hline Single drawing entrance & 33.553 \\
Two symmetric drawing entrances & 41.948 \\
Two asymmetric drawing entrances & 39.654 \\
Three drawing entrances & 36.259 \\
Four drawing entrances & 37.215 \\
\hline
\end{tabular}

cement-sand ratio of $1: 2.3$, and their compressive strength is $35 \mathrm{MPa}$.

4.2.1. Benchmark Experiments. Figures $6 \sim 8$ show the erosion performance comparison of premixed abrasive water, pulsed premixed abrasive water jet, and pulsed abrasive entrained water jet. It can be seen that the rock-breaking performance of the pulsed abrasive water jet is much better than that of the premixed abrasive water jet. In a relatively low concentration, the rock-breaking performance of the pulsed abrasive entrained water jet is very close to that of the premixed pulsed abrasive water jet. But, when the abrasive concentration is higher than $5 \%$, the erosion volume of the pulsed premixed abrasive water jetis gradually larger than that of the pulsed abrasive entrained water jet. As in low concentration, it needs little energy consumption to draw per unit of volume abrasive-containing fluid into the internal mixing chamber, so the abrasive particles are easier to be mixed into the water jet and enter the central part of it. Then, the mixed abrasive water jet will be accelerated by the bit nozzles and will impact on the rocks. But, when the 


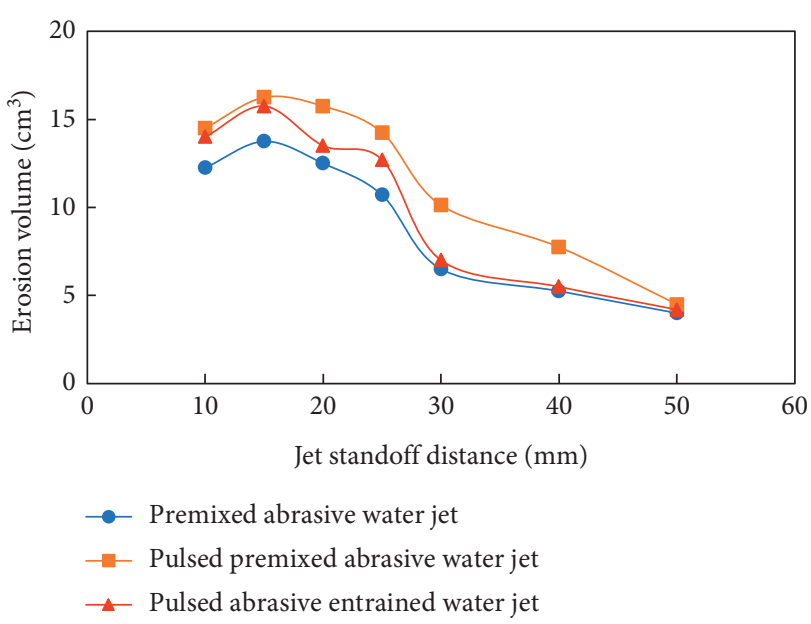

FIGURE 6: Erosion performance of the three kinds of abrasive water jets (when the pump pressure is $15 \mathrm{MPa}$ and the abrasive concentration is $1 \%$ ).

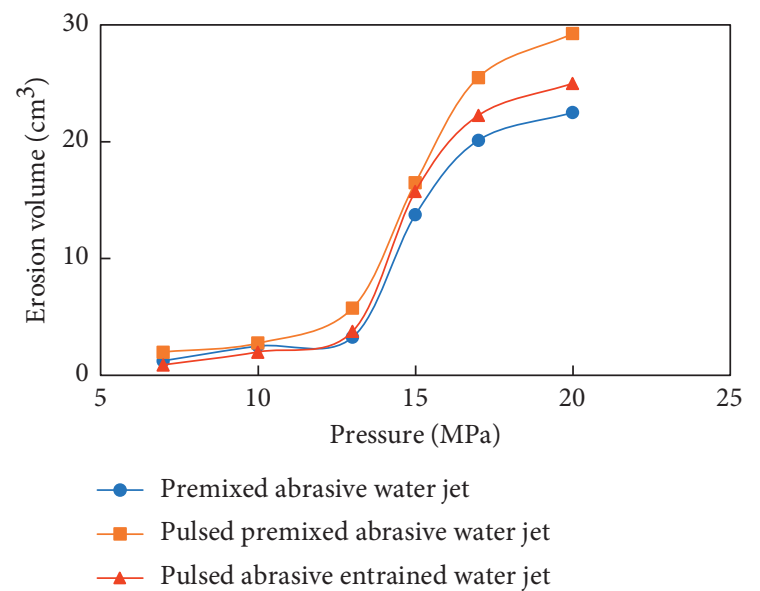

FIGURE 7: Erosion performance of the three kinds of abrasive water jets (when the jet standoff distance is $15 \mathrm{~mm}$ and the abrasive concentration is $1 \%$ ).

concentration is higher, it needs more energy consumption to draw per unit of volume abrasive-containing fluid into the internal mixing chamber. In this way, the abrasive particles are more difficult to be mixed into the water jet, so this process consumes much kinetic energy. As the abrasive particles are well mixed with the water in the long pipeline, the premixed abrasive water jet is mixed much better than the abrasive entrained water jet and has a higher kinetic energy $[25,26]$.

4.2.2. Experimental Factors. The erosion volume first increases and then decreases with the increase in the jet standoff distance (Figure 6). The reason is that when the jet standoff distance is shorter, the pulsed abrasive water jet will not fully develop, so the impact area becomes smaller; the impact jet and the return jet after impacting the rock can interfere with each other strongly, resulting in a greater energy consumption; when

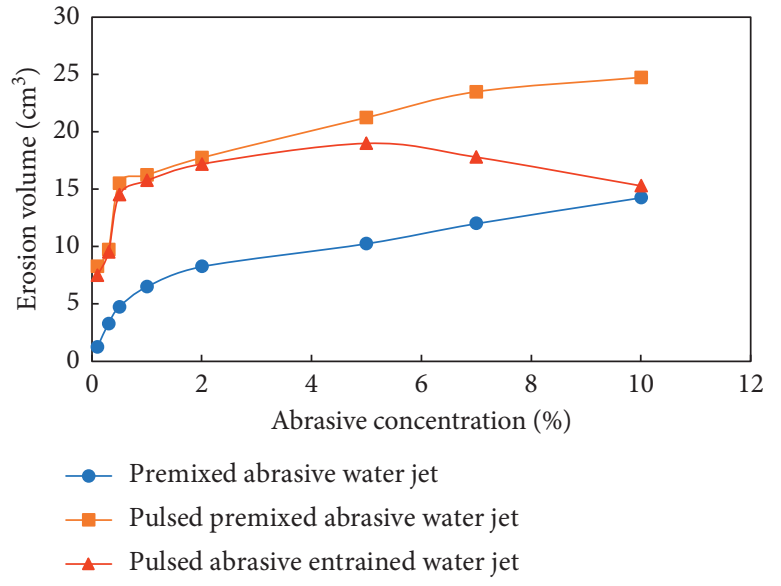

FIGURE 8: Erosion performance of the three kinds of abrasive water jets (when the pump pressure is $15 \mathrm{MPa}$ and the jet standoff distance is $15 \mathrm{~mm}$ ).

the jet standoff distance increases appropriately, the jet turns to fully develop, so the impact area and volume erosion will increase; when the jet standoff distance goes on increasing, the energy consumption will increase rapidly and meanwhile the impact strength and the erosion volume will reduce correspondingly. The best jet standoff distance in the experimental conditions is $15 \mathrm{~mm}$.

Figure 7 shows that the erosion performance improves with the increase in pump pressure because at this moment, the abrasive particles are accelerated in the nozzles, so the impact energy increases, as well as the breaking performance of the water jet on the rocks. When the pump pressure is higher than $15 \mathrm{MPa}$, the erosion volume increases very fast; however, the increasing tendency slows down gradually when the pressure is over $15 \mathrm{MPa}$. Therefore, the operation pressure selected in the experiment is $15 \mathrm{MPa}$.

The erosion volume first increases and then decreases with the increase in the abrasive concentration (Figure 8). Because the bigger the abrasive concentration is, the more abrasive particles there are, which improves the rock-breaking performance. However, as the pulse amplitude of the pulsed abrasive water jet decreases, the total kinetic energy will reduce, and at the same time, the collision probability among the abrasive particles increases, resulting in more energy consumption. When the concentration is less than $5 \%$, the amount of abrasive will play a great role in the erosion performance of the abrasive water jet. A slight decline in the rate of abrasive will affect little on the erosion performance. When the abrasive concentration increases to $5 \%$ or even more than $5 \%$, the kinetic energy of the abrasive particles will decline largely, and the erosion performance will reduce with the increase in the abrasive concentration.

It can be seen that the size range of $0.4 \mathrm{~mm} \sim 0.8 \mathrm{~mm}$ of quartz sand has a best rock-breaking performance in the same concentration (Table 4). The reason is that at a certain impact speed, the kinetic energy as well as the breaking performance improves with the increase in particle size below a certain critical size. However, the energy consumption and erosion performance reduce together with the further increase in particle size above the critical value. 
TABLE 4: Effect of abrasive grain size on the erosion performance.

\begin{tabular}{lc}
\hline Size range of quartz sand $(\mathrm{mm})$ & Erosion volume $\left(\mathrm{cm}^{3}\right)$ \\
\hline $0.3 \sim 0.6$ & 9.5 \\
$0.4 \sim 0.8$ & 15.75 \\
$0.6 \sim 1.2$ & 5 \\
\hline
\end{tabular}

TABLE 5: Effect of abrasive types on the erosion performance.

\begin{tabular}{lc}
\hline Abrasive type & Erosion volume $\left(\mathrm{cm}^{3}\right)$ \\
\hline $1 \%$ quartz sand & 15.750 \\
$1 \%$ garnet & 19.688 \\
$1 \%$ corundum & 27.563 \\
\hline
\end{tabular}

From Table 5, we can see that erosion performance of the high hardness particles such as corundum is much better than that of quartz sand. However, the high hardness materials will also cause serious wear to the drilling tool and the pipeline, so we should comprehensively consider the drilling speed and tool life and then select the reasonable abrasive medium. In contrast, choosing quartz sand as the abrasive medium can reduce the wear of drilling tools and obtain certain rock-breaking efficiency, which is suitable for drilling in deep oil and gas reservoirs.

\section{Conclusions}

(1) The experiment results show that the rock-breaking performance of the pulsed water jet is much better than that of the continuous water jet. While the fluid entrance is open and the external water is drawn in, the rock-breaking performance of the pulsed water jet will be improved significantly.

(2) The rock-breaking performance of the pulsed abrasive water jet is much better than that of the premixed abrasive water jet. In a relatively low concentration, the rock-breaking performance of the pulsed abrasive entrained water jet is very close to that of the premixed pulsed abrasive water jet.

(3) The experiment of the pulsed abrasive entrained water jet has gained a very good rock-breaking performance. The above research lays a foundation for the application of the pulsed abrasive water jet in the development of deep oil and gas reservoirs.

\section{Data Availability}

The data used to support the findings of this study are included within the article.

\section{Conflicts of Interest}

The authors declare that they have no conflicts of interest regarding the publication of this paper.

\section{Acknowledgments}

This study was supported by the Fundamental Research Funds for the Central Universities of China (18CX02072A) and the Opening Fund of Key Laboratory of Mining Disaster Prevention and Control (MDPC201908). The authors would like to acknowledge the valuable contributions of their colleagues and the support of China University of Petroleum for the preparation of this paper.

\section{References}

[1] H. Wang, Y. Ge, and L. Shi, "The present situation, challenges and development direction of ultra-deep well drilling and completion technology in deep well and ultra-deep well," Natural Gas Industry, vol. 37, pp. 1-8, 2017.

[2] Z. H. Shen, Water Jet Technology in Petroleum Engineering, Petroleum University Press, Dongying, China, 1997.

[3] Z. H. Shen, Theory and Technique of Water Jet, China University of Petroleum Press, Dongying, China, 1998.

[4] Y. Lu, J. Tang, Z. Ge, B. Xia, and Y. Liu, "Hard rock drilling technique with abrasive water jet assistance," International Journal of Rock Mechanics and Mining Sciences, vol. 60, pp. 47-56, 2013.

[5] W. J. Kogelmann, E. D. Thimons, J. E. Virgona, and L. A. Weakly, "Water-jet-assisted mechanized oil shale mining factory test," in Proceedings of the Oil Shale Symposium, Colorado School of Mines, Golden, CO, USA, pp. 5677, April 1989.

[6] L. Bai, L. Zhou, X. Jiang, Q. Pang, and D. Ye, "Vibration in a multistage centrifugal pump under varied conditions," Shock and Vibration, vol. 2019, Article ID 2057031, 9 pages, 2019.

[7] F. Ren, T. Fang, and X. Cheng, "Study on rock-breaking depth and damage area under particle jet impact," Shock and Vibration, vol. 2020, Article ID 4140165, 12 pages, 2020.

[8] Z. H. Shen, G. S. Li, and R. Wang, "Application and prospects of water jet technology in petroleum engineering," Engineering Science, vol. 12, no. 4, pp. 60-64, 2002.

[9] R. Kovacevic, R. Mohan, M. Ramulu et al., "State of the art of research and development in abrasive waterjet machining," ASME Journal of Manufacturing Science and Engineering, vol. 119, 1997.

[10] J. S. Cobbett, "Sand jet perforating revisited," in Proceedings of the SPE 39597, Denver, CO, USA, April 1998.

[11] J. J. Kolle, "Increasing drilling rate in deep boreholes by impulsive depressurization," in Proceedings of the Fourth North American Rock Mechanics Symposium, Seattle, WA, USA, July 2000.

[12] D. Arola, A. E. Alade, and W. Weber, "Improving fatigue strength of metals using abrasive waterjet peening," $M a$ chining Science and Technology, vol. 10, no. 2, pp. 197-218, 2006.

[13] H. Li, R. Wang, D. Yang, W. Zhou, and L. Li, "Determination of rotary cutting depth on steel pipes with the abrasive water jet technique," Proceedings of the Institution of Mechanical Engineers, Part C: Journal of Mechanical Engineering Science, vol. 225, no. 7, pp. 1626-1637, 2011.

[14] L. Zhou, Y. J. Gong, Z. M. Zhang, and Z. W. Wang, "Software technology research on control system of ultra high pressure water jet rust removal equipment," Advanced Materials Research, vol. 721, pp. 372-376, 2013.

[15] Y. Yang, W. Li, L. Zhou, and W. Zhang, "Experimental study on the unsteady characteristics and the impact performance of 
a high-pressure submerged cavitation jet," Shock and Vibration, vol. 2020, Article ID 1701843, 15 pages, 2020.

[16] W. Q. Tao, Numerical Heat Transfer Theory, Jiaotong University Press, Shanghai, China, 2001.

[17] M. B. Wang and R. H. Wang, "Application of K-E models on the numerical simulation of a semi-confined turbulent impinging jet," in Proceedings of the 8th Pacific Rim International Conference on Water Jet Technology, Qingdao, China, October 2005.

[18] Z. W. Wang, B. Chen, and L. J. Guo, "Numerical simulation of motion and bubble entrainment of vortex ring," Journal of Engineering Thermophysics, vol. 29, no. 4, pp. 613-616, 2008.

[19] L. Q. Wang, X. M. Wang, and R. L. Xu, "Experimental study on structural parameters optimized design of the self-excited oscillation pulsed jet nozzle," Journal of Engineering Thermophysics, vol. 25, no. 6, pp. 956-958, 2004.

[20] K. Babets and E. Geskin, "Numerical study of the turbulent flow inside a pure waterjet," in Proceedings of the 2001 WJTA American Waterjet Conference, Minneapolis, Minnesota, August 2001.

[21] D. H. Ahmed, E. Siores, and F. L. Chen, "Numerical simulation of abrasive water jet," in Proceedings of the 2001 WJTA American Waterjet Conference, Minneapolis, Minnesota, August 2001.

[22] H. J. Ni, L. J. Han, and R. H. Wang, “Analyze the assistant rock-breaking mechanism of bottom-hole jet of 3-nozzle 3cone bit," Natural Gas Industry, vol. 26, no. 6, pp. 72-74, 2006.

[23] J. Y. Xiong, J. H. Fu, and S. H. Qian, "Mechanism of oscillating jet induced by external excitation," Acta Petrolei Sinica, vol. 5, no. 2, pp. 100-102, 2004.

[24] H. J. Ni, Y. K. Du, and R. H. Wang, "Study on the lab experiment of pulsed abrasive water jet using bottom hole cuttings," Oil Drilling \& Production Technology, vol. 30, no. 5, pp. 25-28, 2008.

[25] M. B. Wang and R. H. Wang, "Advances in the process control of abrasive water jet," Lubrication Engineering, vol. 6, pp. 204-207, 2005.

[26] M. B. Wang and R. H. Wang, "Numerical simulation on fluidpartical two-phase jet flow field in nozzle," Journal of China University of Petroleum (Edition of Natural Science), vol. 29, no. 5, pp. 46-49, 2005. 